\title{
Optically induced flat bands in twisted bilayer graphene
}

\author{
Or Katz, $, 2,3,{ }^{*}$ Gil Refael, ${ }^{4}$ and Netanel H. Lindner ${ }^{1}$ \\ ${ }^{1}$ Physics Department, Technion, Haifa 320003, Israel \\ ${ }^{2}$ Rafael Ltd., Haifa 3102102, Israel \\ ${ }^{3}$ Department of Physics of Complex Systems, Weizmann Institute of Science, Rehovot 76100, Israel \\ ${ }^{4}$ Department of Physics, Institute of Quantum Information and Matter, California Institute of Technology, Pasadena, California 91125, USA
}

(Received 19 November 2019; revised 7 September 2020; accepted 14 September 2020; published 16 October 2020)

Twisted bilayer graphene at the magic twist angle features flat energy bands, which lead to superconductivity and strong correlation physics. These unique properties are typically limited to a narrow range of twist angles around the magic angle with a small allowed tolerance. Here, we report on a mechanism that enables flattening of the band structure using coherent optical illumination, leading to emergence of flat isolated Floquet-Bloch bands. We show that the effect can be realized with relatively weak optical beams at the visible-infrared range (below the material bandwidth) and persist for a wide range of small twist angles, increasing the allowed twist tolerance by an order of magnitude. We discuss the conditions under which these bands exhibit a nonzero Chern number. These optically induced flat bands could potentially host strongly correlated nonequilibrium electronic states of matter.

DOI: 10.1103/PhysRevB.102.155123

\section{INTRODUCTION}

Van der Waals heterostructures are a prominent tool for discovery of emergent phenomena in condensed-matter physics. These materials allow for a considerable degree of control in their physical structure, being formed by stacking of individual atomic layers [1-3]. Stacking different atomic layers with a relative angular twist has become a salient mechanism in structuring the energy bands of these materials [4-7]. This twist forms a slowly varying moiré pattern, which modulates the interlayer electronic potential. At certain twist angles, the bands near the charge-neutrality point (CNP) can become flat and relatively isolated from other bands [8-11]. These flat bands have recently attracted considerable attention with the discovery of superconductivity, correlated insulating states, and ferromagnetism, which emerge at low temperatures [12-17].

Twisted bilayer graphene (TBG) exhibits isolated flat bands when twisted near the magic twist angle $\theta_{\mathrm{m}} \approx 1.1^{\circ}$. At smaller twist angles $\theta<\theta_{\mathrm{m}}$, a larger moiré pattern is formed, eliminating the energy gap to distant energy levels and increasing the bandwidth of the bands near the CNP $[9,18]$. Therefore, the discovery of strong correlated phenomena has been limited to a small range of twist angles near the magic angle where the band-structure features narrow gaped bands.

Floquet engineering with optical fields is a valuable technique that could induce topological band structures and electronic correlations in various materials [19-35]. Floquet engineering of TBG has been considered as a technique to tune the value of the magic angle using longitudinal waves [36], and to control the topology of the bands near the CNP at large twist angles [37]. However, the idea of significantly

*Corresponding author: or.katz@ weizmann.ac.il reducing the bandwidth of certain bands in the band structure generating flat bands has never been realized in Van der Waals heterostructures with optical fields. Although the mechanism of dynamic localization could be theoretically applied [38], it practically requires extremely high fields which render it unfeasible.

Here, we consider small-angle twisted bilayer graphene driven with optical fields as shown in Fig. 1. We demonstrate that a driving laser could improve the flatness of the bands near the CNP even at twist angles smaller than the magic angle. We further show that the driving field opens a gap between the emerging flat bands, increases the gaps separating them from other bands, and could induce a nontrivial Berry curvature. The presented effect is found robust for lattice relaxation and could potentially be implemented with relatively weak sub-bandwidth optical fields in the visible range.
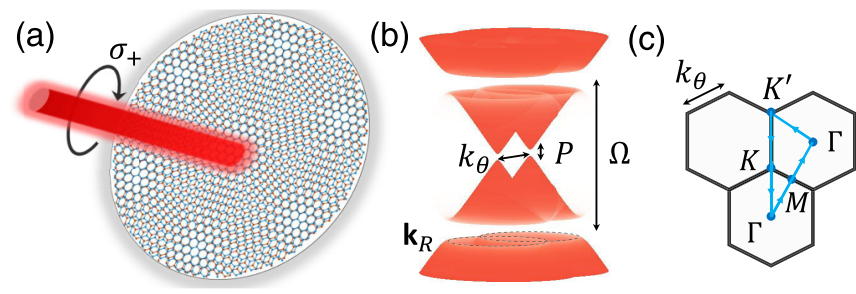

FIG. 1. Schematics of Floquet twisted bilayer graphene. (a) TBG driven by a circularly polarized optical laser field. (b) Illustration of the band structure of optically driven TBG with zero interlayer coupling near the $K$ points of the two layers. The laser frequency $\Omega$ in the visible range is much smaller than graphene bandwidth, opening a photoinduced gap $P$ at the $K$ points of the two layers whereas avoiding side-band transitions near these points. Nonzero interlayer hopping leads to hybridization of the Dirac cones. (c) Moiré Brillouin zone with the trajectory $K^{\prime} \rightarrow K \rightarrow \Gamma \rightarrow M \rightarrow \Gamma \rightarrow K^{\prime}$. 


\section{MODEL}

We model the low-energy band structure of TBG using a continuum model for a single valley and spin [9,10,39-43]. These models, accurately describe the Hamiltonian of TBG with a relatively small twist angle $\left(\theta \lesssim 10^{\circ}\right)$ where intervalley processes are strongly suppressed. We focus our discussion on a simple model which has relatively few parameters, and verify our results with a detailed model that accurately treats lattice reconstruction effects which is presented in Sec. VI. In the absence of a driving field, our model Hamiltonian is given by

$$
H=\left(\begin{array}{cc}
h(\theta / 2, \mathbf{r}) & T(\mathbf{r}) \\
T^{\dagger}(\mathbf{r}) & h(-\theta / 2, \mathbf{r})
\end{array}\right)
$$

which acts on the spinor $\Psi(\mathbf{r})=\left(\psi_{1 \mathrm{~A}}, \psi_{1 \mathrm{~B}}, \psi_{2 \mathrm{~A}}, \psi_{2 \mathrm{~B}}\right)^{T}$. The subscripts 1 and 2 denote the top and bottom layers, respectively, and the $A, B$ subscripts denote the sublattice isospin of a monolayer. The Hamiltonians of the two rotated monolayers of graphene are denoted by $h( \pm \theta / 2, \mathbf{r})$, and feature a nearestneighbor coupling with a hopping amplitude $\tau$. The operator $T(\mathbf{r})$ denotes the periodic interlayer moiré potential,

$$
T(\mathbf{r})=\sum_{n=1}^{3}\left[w_{0} \sigma_{0}+w_{1}\left(\sigma_{x} \cos n \phi+\sigma_{y} \sin n \phi\right)\right] e^{i\left(n \phi-\phi-\mathbf{q}_{n} \mathbf{r}\right)} .
$$

We use the standard Bernal stacking for untwisted layers $(\theta=0)$ and $\phi=2 \pi / 3$. The set of wave-numbers $\mathbf{q}_{1}=$ $k_{\theta}(0,-1), \mathbf{q}_{2,3}=k_{\theta}( \pm \sqrt{3}, 1) / 2$, represents the relative displacements of the Dirac cones between the layers where $k_{\theta}=4 \pi \theta /(3 \sqrt{3} a)$ is determined by the twist angle $\theta$ and $a=1.42 \AA$. The $2 \times 2$ Pauli matrices and identity matrix are denoted by $\sigma$ and $\sigma_{0}$, respectively. $w_{0}$ denotes the interlayer coupling between the $A A$ and $B B$ domains, and $w_{1}$ denotes the $A B$ and $B A$ interlayer coupling. Our model uses the exact band structure of monolayer graphene (generalizing approaches using the $k p$ approximation) for better modeling of the higher-energy levels. We use $\tau=2.73 \mathrm{eV}, w_{1}=$ $110 \mathrm{meV}$, and account for the effects of lattice relaxation by approximating $w_{0}=0.8 w_{1}[10,18,40,41]$. The lattice relaxation parameter $w_{1} / w_{0}$ is, in fact, the leading term of the detailed model in Sec. VI.

We consider a circularly polarized driving light field of frequency $\Omega$, represented by the electric-field $\mathcal{E}(t)=$ $\mathcal{E}[\cos (\Omega t) \hat{x}-\sin (\Omega t) \hat{y}]$. We take the light field to be at normal incidence and uniform over the sample. We model the interaction with the driving field using a Peierls substitution for the intralayer hopping parameters in the Hamiltonian, $\tau \rightarrow$ $\tau \exp (-i e \mathcal{E} a / \hbar \Omega)$. In the presence of the time-periodic drive, the solution of the Schrödinger equation can be indexed by the quasienergies $\varepsilon$, which fall within a single "Floquet-Brillouin" zone $-\hbar \Omega / 2 \leqslant \varepsilon<\hbar \Omega / 2$, and can be written as

$$
\left|\psi_{v}(t)\right\rangle=e^{-i \varepsilon_{v} t / \hbar} \sum_{m=-\infty}^{\infty} e^{-i m \Omega t}\left|\psi_{v}^{(m)}\right\rangle,
$$

where the index $v$ carries all other quantum numbers of the state. The set of modes $\sum_{m}\left|\psi_{v}^{(m)}\right\rangle$ are the eigenmodes of the Floquet Hamiltonian which we numerically solve in momentum-space, truncating both the number of Floquet
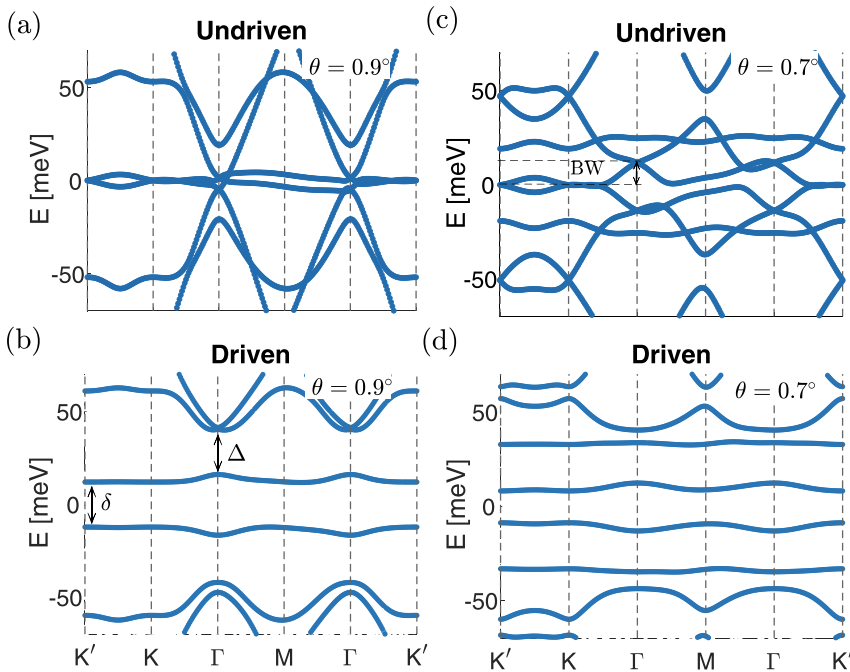

(d)

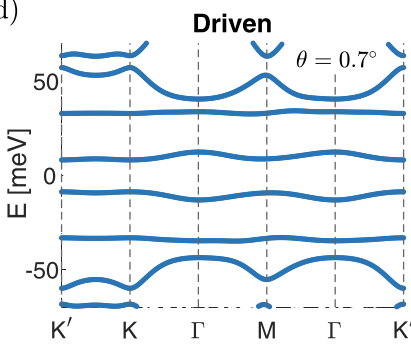

FIG. 2. Floquet band structure of TBG below the magic angle for the simple model. The band structure shown corresponds to $\theta=0.9^{\circ}$ and $\theta=0.7^{\circ}$ (the first magic angle is at $\theta_{\mathrm{m}} \approx 1.1^{\circ}$ ) and are plotted along the contour shown in Fig. 1(c). (a) and (c) Undriven TBG. At the charge neutrality point, level crossing at the $\Gamma$ point increases the effective bandwidth of the two bands near $E=0$, and no isolated flat bands are observed. (b) and (d) Optically driven TBG. The upper and lower bands near $E=0$ become gapped, resulting with nearly flat bands. In panels (b) and (d), we plot the time-averaged density of states $\bar{\rho}_{0}(E)$, see Eq. (3), for a driving field of frequency $\hbar \Omega=$ $1.5 \mathrm{eV}$ and peak electric field of $\mathcal{E}=5.6 \mathrm{MV} / \mathrm{cm}$. Only Floquet states with significant spectral weight $\left[A_{v}^{0}(\mathbf{k})>0.05\right.$, cf. Eq. (3) $]$ are shown.

blocks and the infinite representation of the matrix $T(\mathbf{k})$, see Appendix A.

\section{FLOQUET FLAT BANDS}

Typical Floquet band structures of TBG are shown in Figs. 2(a)-2(d), in the presence and absence of a driving field. The band structures are plotted along a contour in the first moiré Brillouin zone $(\mathrm{mBz})$, which is a hexagon with size $k_{\theta}$ as shown in Fig. 1(c). For the undriven case of TBG with twist angle $\theta=0.9^{\circ}$, the lower and upper bands near $E=0$ experience level crossing with other bands at the $\Gamma$ point shown in Fig. 2(a). The large bandwidth of the resulting connected group of bands manifests larger kinetic energy of the electrons which hinders the observation of strong correlation effects. Upon driving, an energy gap $\delta$ between the lower and the upper bands is opened, as well as an energy gap $\Delta$ isolating these two bands from the rest of the spectrum. These gaps are shown in Fig. 2(b) for a drive with $\hbar \Omega=1.5 \mathrm{eV}$ and peak electric field of $\mathcal{E}=5.6 \mathrm{MV} / \mathrm{cm}$. For $\theta=0.7^{\circ}$, the undriven band structure exhibits larger bandwidth and multiple level crossings as shown in Fig. 2(c). The drive opens the energy gaps $\delta$ and $\Delta$ and decreases the bandwidth of the lower and upper bands, thus, flattening the bands as shown in Fig. 2(d). Interestingly, here, the drive also flattens the next-nearest bands to CNP.

To quantify the effect of the drive on the band structure, in Figs. 3(a)-3(c), we plot the gaps and bandwidth of the upper 

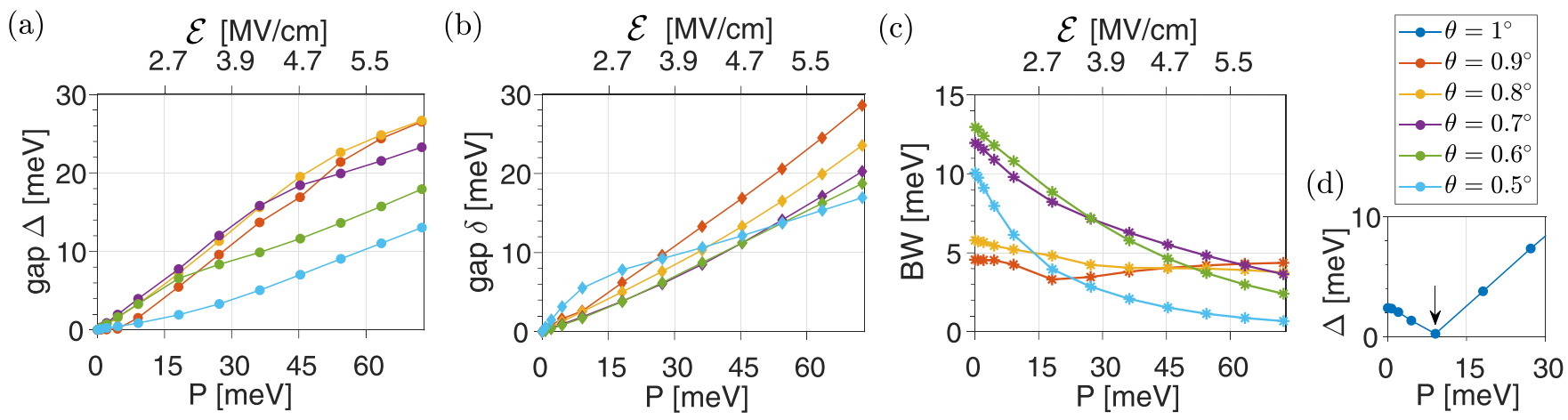

FIG. 3. Characteristics of the upper Floquet band near the charge-neutrality point at $\hbar \Omega=1.5 \mathrm{eV}$ and angles $\theta \leqslant 1^{\circ}$ below the first magic angle for the simple model. (a) Increase in the gap $\Delta$ to remote bands with increasing photoinduced gap $P$ (proportional to the optical intensity), which isolates the flat bands. $\mathcal{E}$ denotes the peak electric field of the drive. (b) The gap $\delta$ between the upper and the lower bands at the charge-neutrality point increases as a function of $P$. (c) Narrowing of the bandwidth (BW) with increasing $P$ at $\theta \leqslant 0.8^{\circ}$. (d) Closing of the gap $\Delta$ near $P=10 \mathrm{meV}$ for $\theta=1^{\circ}$ is associated with change in the topology of the flat band. In all panels, the quantities were calculated from the time-averaged density of states $\bar{\rho}_{0}(E)$, see Eq. (3), including only Floquet states with significant spectral weight $A_{v}^{0}(\mathbf{k})>0.05$.

Floquet band at $\hbar \Omega=1.5 \mathrm{eV}$ as a function of the twist angle $\theta$ and the quantity $P=(3 \tau e a \mathcal{E})^{2} /\left(2 \hbar^{3} \Omega^{3}\right)$, which gives the photoinduced gap in monolayer graphene (and is proportional to the intensity of the drive at a given frequency) [21,23]. In the absence of a drive, the energy gap $\delta$ between the lower and the upper bands vanishes due to the symmetries of monolayer graphene, and the gap $\Delta$ isolating these two bands vanishes for $\theta \leqslant 0.9^{\circ}$ as shown in Figs. 3(a) and 3(b). Upon irradiation, for $\theta \leqslant 0.9^{\circ}$, the gaps $\Delta$ and $\delta$ obtain nonzero values and increase almost linearly with $P$, thus, yielding isolated, narrow bands. In Fig. 3(c), we plot the bandwidth of the upper Floquet band $\varepsilon_{\text {up }}(\mathbf{k})$ given by $\mathrm{BW}=\max \left[\varepsilon_{\text {up }}(\mathbf{k})\right]-\min \left[\varepsilon_{\text {up }}(\mathbf{k})\right]$ for $\mathbf{k}$ in the mBz. For $\theta \leqslant 0.8^{\circ}$, the bandwidth of the upper band decreases as the amplitude of the drive is increased. We, thus, conclude that light irradiation allows for emergence of narrow isolated bands at twist angles smaller than the magic angle.

\section{DEPENDENCE ON THE DRIVE FREQUENCY}

The Floquet band structure of TBG in the presence of the drive is a result of both on-resonant and off-resonant processes. Since the number of bands in the reduced $\mathrm{mBz}$ for any quasimomentum is extremely large, it is important to demonstrate that the interaction with the drive, especially at frequencies below the bandwidth of the material, does not mix the low-energy bands with high-energy bands. We, therefore, demonstrate that the presented mechanism is associated with an off-resonant process and identify the range of frequencies in which the hybridization of the resulting flat bands with high-energy bands is suppressed.

\section{A. Numerical analysis}

To this effect, we define the time-averaged density of states (DOS) defined as [35]

$$
\bar{\rho}_{0}(\mathbf{k}, E)=\sum_{v} \sum_{m} A_{v}^{(m)}(\mathbf{k}) \delta\left(\varepsilon_{v}+m \hbar \Omega-E\right),
$$

with $A_{v}^{(m)}(\mathbf{k})=\left|\psi_{v}^{(m)}(\mathbf{k})\right|^{2}$. The DOS $\bar{\rho}_{0}(\mathbf{k}, E)$ is the imaginary part of the time-averaged Green's function [24,35,44]. To quantify the sharpness of the bands near $E=0$, we integrate $\bar{\rho}_{0}(\mathbf{k}, E)$ in a small interval $\Delta E=40 \mathrm{meV}$ and compute the total intensity $I(\mathbf{k})=\int_{-\Delta E / 2}^{\Delta E / 2} d E \bar{\rho}_{0}(\mathbf{k}, E)$ at a given momentum. $I(\mathbf{k})$ consists of discrete contributions from Floquet bands with $2\left|\varepsilon_{v}\right| \leqslant \Delta E$, written as $I(\mathbf{k})=\sum_{v} I_{v}(\mathbf{k})$. In Figs. 4(a)-4(c), we plot the distribution of $I_{v}=I_{v}(\mathbf{k}) d k$ where the integral follows the contour in Fig. 1(c). We compare the histograms of $I_{v}$ for different drive frequencies at $\theta=0.9^{\circ}$, maintaining the gaps $\Delta(\Omega)$ and $\delta(\Omega)$ constant throughout the three panels by keeping the photoinduced gap constant with $P=33 \mathrm{meV}$.

For drive frequencies in the UV-visible range, the upper and lower flat bands are sharp. This is indicated by the distribution of $I_{v}$ which has a sharp peak at $I=1$ corresponding to the two flat bands and no other weights except at $I \approx 0$. Such a distribution is shown in Fig. 4(a), for a driving field in the visible range $(\hbar \Omega=3 \mathrm{eV})$. For a near-infrared driving field, the spectral weight of the flat bands is reduced whereas background spectral weight and band crossings appear as shown in Fig. 4(d) in which we plot $\bar{\rho}_{0}(\mathbf{k}, E)$ at $\hbar \Omega=0.94 \mathrm{eV}$. These effects lead to the broad distribution of $I_{v}$ shown in Fig. 4(b) with many Floquet eigenstates corresponding to $0<I_{v}<0.2$ and the two flat Floquet bands corresponding to $I_{v}>0.2$ at most momenta (recall that the distributions $I_{v}$ are averaged along the contour in the $\mathrm{mBz}$ ). The band structure in Fig. 4(d) can be compared with the sharply defined bands in Fig. 2 which shows $\bar{\rho}_{0}(\mathbf{k}, E)$ at $\hbar \Omega=1.5 \mathrm{eV}$. At even lower driving frequencies, the DOS is dominated by rapidly oscillating Floquet bands with low spectral weight at the $|E| \leqslant \Delta E / 2$ spectral window as implied by $I_{v}$ approaching a Poisson distribution, shown in Fig. 4(c) for $\hbar \Omega=0.3 \mathrm{eV}$.

To estimate the range of frequencies for which the DOS at energies $|E| \leqslant \Delta E / 2$ is predominately sharp, we consider the quantity,

$$
\mathcal{S}_{n}=\oint\left(\sum_{v}\left[I_{v}(\mathbf{k})\right]^{n} / \sum_{v} I_{v}(\mathbf{k})\right) d k,
$$

along the contour in Fig. 1(c). For $n \geqslant 2, \mathcal{S}_{n}$ approaches unity for a fully sharp DOS, i.e., when the distributions $I_{v}(\mathbf{k})$ are bimodal and peaked at $I=0$ or $I=1$ at all k's. Conversely, $\mathcal{S}_{n}$ with $n \geqslant 2$ becomes vanishingly small when $I_{v}(\mathbf{k})$ 
(a)

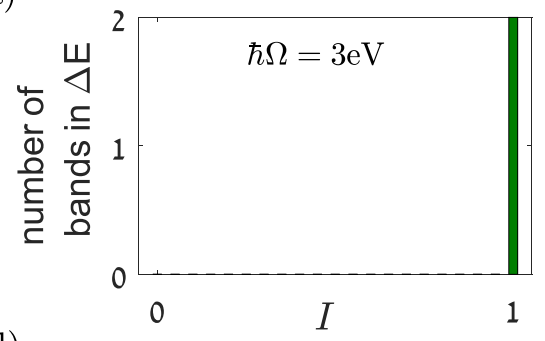

(d)

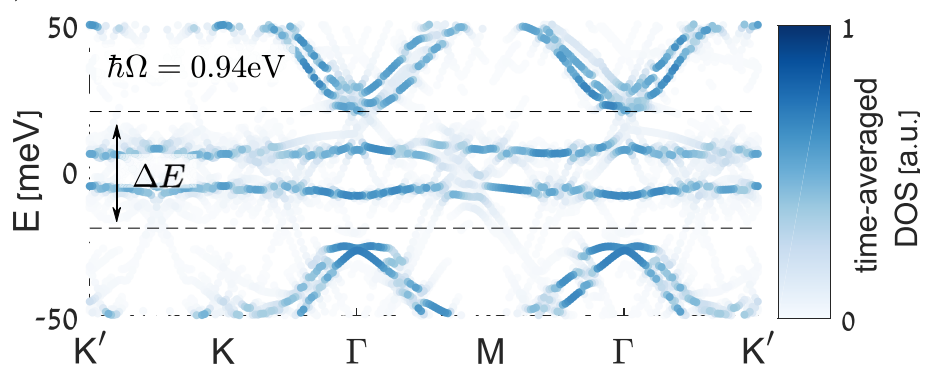

(b)

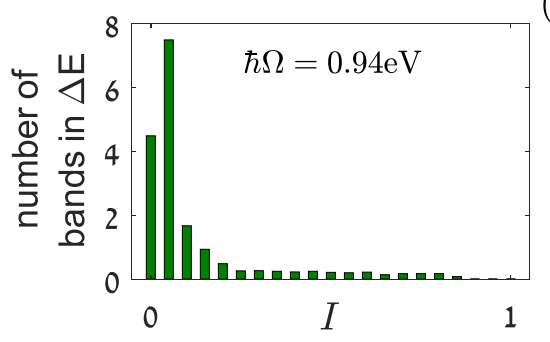

(c)

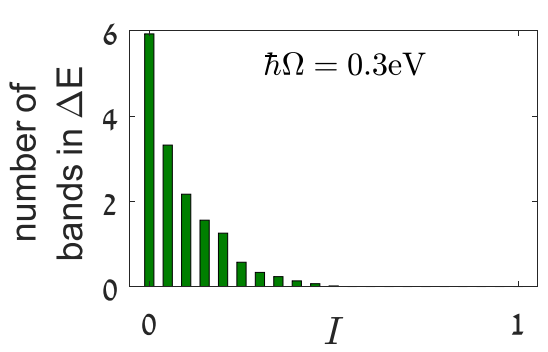

FIG. 4. Mixing with higher-energy bands at low driving frequencies. (a)-(c) Distributions of $I_{v}$, characterizing the time-averaged DOS at energies $|E| \leqslant 20 \mathrm{meV}$, averaged along the contour $K^{\prime} \rightarrow K \rightarrow \Gamma \rightarrow M \rightarrow \Gamma \rightarrow K^{\prime}$ for $\theta=0.9^{\circ}$ and constant photoinduced gap $P=$ $33 \mathrm{meV}$ in the simple model. (a) For a high drive frequency $\hbar \Omega=3 \mathrm{eV}$, the time-averaged DOS in the energy interval $|E| \leqslant 20 \mathrm{meV}$ is sharp, yielding a bimodal distribution peaked at $I=1$ and $I=0$ (not shown). (b) and (c) For lower frequencies $\hbar \Omega=0.94,0.3 \mathrm{eV}$ the number of bands with significant spectral weight increases, indicating mixing with higher-energy bands. (d) The time-averaged DOS at $\hbar \Omega=0.94 \mathrm{eV}$, which shows mixing with higher energy bands. (e) Characterization of the sharpness of the time-averaged DOS as a function of drive-frequency. At drive frequencies in the UV-NIR range $\hbar \Omega \gtrsim 1.2 \mathrm{eV}$, the sharpness parameter $\mathcal{S}_{4}$ approaches unity, indicating that the interaction with the drive is predominately off-resonant.

takes the Poisson form peaked at $I=0$ as in Fig. 4(c). In Fig. 4(e), we plot $\mathcal{S}_{4}(\Omega)$, finding that the DOS remains sharp for driving frequencies $\Omega \gtrsim \Omega^{*}$ with $\hbar \Omega^{*}=1.2 \mathrm{eV}$, which contains the visible range down to frequencies which are significantly smaller than the graphene bandwidth $\sim 17 \mathrm{eV}$. At drive frequencies in the infrared and below, $\Omega \lesssim \Omega^{*}$, the DOS becomes smeared.

\section{B. Analytical estimation}

To understand the threshold frequency $\Omega^{*}$, we analyze the mechanism that decreases $\mathcal{S}_{4}$ for weak interlayer coupling, $\alpha=\left(w_{0}+w_{1}\right) /\left(3 t k_{\theta}\right) \ll 1$ and weak driving $P \ll \hbar \Omega$. When the interlayer coupling is absent $(\alpha=0)$, the Floquet band structure is that of two driven graphene monolayers as shown in Fig. 1(b). Note that the Floquet band structure in Fig. 1(b) exhibits contours of resonant momenta $\mathbf{k}_{R}$ which encircle the $K$ points of both monolayers and for which the energy difference between the conduction and the valence bands is equal to $\Omega$. At momenta which are deep within this contour (for which $\left|\varepsilon_{v}\right| \ll \hbar \Omega / 2$ ), the expansion of the Floquet states according to Eq. (2) is dominated by the $m=0$ component. At the resonant momenta, the original valence and conduction bands with energies $E_{\mathrm{v}}(\mathbf{k})$ and $E_{\mathrm{c}}(\mathbf{k})$ are strongly mixed, and, therefore, the Floquet states consist of both $m=0$ and $m=1$ (or $m=-1$ ) components with similar amplitudes. Moving away from the resonant momenta, the square of the amplitude of the $m= \pm 1$ component, which we denote by $\tilde{\mathcal{N}}(\mathbf{k})$, decreases as a Lorentizan with argument $\left[E_{\mathrm{c}}(\mathbf{k})-E_{\mathrm{v}}(\mathbf{k})-\Omega\right]$ width $\sqrt{\hbar \Omega P}$ and maximum value of $1 / 2$.
We now study the effect of nonzero interlayer coupling, $\alpha>0$. The Floquet-Bloch wave functions of TBG can be written as

$$
|\psi(\mathbf{k}, t)\rangle=\sum_{\nu, n} c_{n, \nu}\left|\tilde{\psi}_{\nu}\left(\mathbf{k}_{\mathbf{n}}, t\right)\right\rangle,
$$

where $|\tilde{\psi}(\mathbf{k}, t)\rangle$ are the Floquet states at $\alpha=0$ and $\mathbf{k}_{n}$ is a discrete set of momenta in the extended mBz (cf. Appendix A). Importantly, the coefficients $c_{n, v}$ of the flat bands decrease as $|\alpha|^{r}$ with $r=\left|\mathbf{k}_{n}\right| / k_{\theta}$ since $r$ interlayer tunneling processes are required to connect Floquet states near the $K$ point with Floquet states at $\mathbf{k}_{n}$. At large drive frequencies, the variation of $I(\mathbf{k})$ along the contour in the $\mathrm{mBz}$ is small since the resonant momenta are outside the first $\mathrm{mBz}$, i.e., $\left|\mathbf{k}_{R}\right| \gg k_{\theta}$. We, therefore, estimate $\mathcal{S}_{4} \approx\left|I_{0}\right|^{3}$ where $I_{0}$ is the contribution to the intensity from one of the Floquet flat bands near the $K$ point. For high frequencies (larger than the graphene bandwidth) the expansion of these states, cf. Eq. (2), is dominated by the $m=0$ component yielding $I_{0} \rightarrow 1$ and $\mathcal{S}_{4} \rightarrow 1$. Lowering the frequency below the graphene bandwidth decreases the magnitude of the resonant momenta $\left|\mathbf{k}_{R}\right|$. This leads to an increase in the amplitudes $c_{n, v}$ corresponding to the resonant momenta in Eq. (5), which, in turn, reduces $I_{0}$.

Using the discussion above, we can estimate the norm of the $m= \pm 1$ component of the upper Floquet flat band as $\mathcal{N}=$ $\sum_{n} \alpha^{2 r\left(\mathbf{k}_{n}\right)} \tilde{\mathcal{N}}\left(\mathbf{k}_{n}\right)$. This component does not contribute to $I_{0}$, and, therefore, $I_{0} \approx(1+\mathcal{N})^{-1}$. Taking the largest contribution in the sum $\mathcal{N}$, we arrive at $I_{0} \approx\left[1+3 r_{*} \alpha^{2 r_{*}} \tilde{\mathcal{N}}\left(r_{*} k_{\theta}\right)\right]^{-1}$ where $r_{*}=\left|\mathbf{k}_{R}\right| / k_{\theta}$, and the factor $3 r_{*}$ arises due to the number of $\mathbf{k}_{n}$ momenta with hopping number $r_{*}$ in the hexagonal grid (see Appendix A). For drive frequencies in the visible 


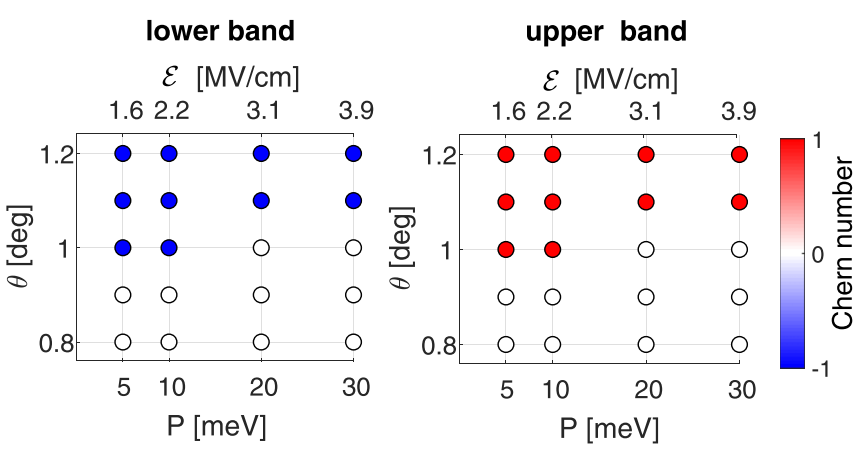

FIG. 5. Chern numbers of flat bands resulting from off-resonant coupling to circularly polarized light. At twist angles $\theta \geqslant \theta^{*} \sim 1^{\circ}$, the lower and upper bands near $E=0$ of TBG with an added Haldane mass term exhibit nonzero Chern numbers when the magnitude $P$ of the mass term is below a critical value. The peak electric-field $\mathcal{E}$ corresponding to $P$ is given for an optical field of frequency $\hbar \Omega=1.5 \mathrm{eV}$

range, we estimate $\left|\mathbf{k}_{R}\right| \approx \hbar \Omega /(3 t a)$ and for $\theta=0.9^{\circ}$ and $P=33 \mathrm{meV}$ we get $\alpha=0.64$. The condition $\mathcal{S}_{4} \approx\left|I_{0}\right|^{3} \geqslant$ 0.5 yields a threshold frequency of $\hbar \Omega^{*} \approx 1 \mathrm{eV}$, which can be compared with the numerically calculated value of $\hbar \Omega^{*}=$ $1.2 \mathrm{eV}$ as shown in Fig. 4(e).

\section{TOPOLOGY OF THE FLAT BANDS}

For drive frequencies higher than the threshold frequency ( $\hbar \Omega \gtrsim 1.2 \mathrm{eV}$ ), a direct coupling between the bands near $\mathrm{CNP}$ and higher levels in the $\mathrm{mBz}$ is suppressed. Thus, the leading-order effect of the interaction with the drive can be approximated with the effective static Hamiltonian $H_{\text {eff }} \approx$ $H+\left[\mathcal{H}_{01}, \mathcal{H}_{10}\right] / \Omega$, describing an off-resonant process which is second order in the drive. The effect of a weak drive $P \ll$ $\hbar \Omega$, can be approximated with an addition of a photoinduced Haldane mass term to the Hamiltonian describing each monolayer. Thus, in this limit, the Hamiltonians $h_{ \pm}(\mathbf{k})$ describing a monolayer in reciprocal space near the $K(+)$ and $K^{\prime}(-)$ points acquire an additional term $\eta P \sigma_{z}$, where $\eta= \pm 1$, respectively. Therefore, the band structure of the driven system can be described as the result of the interlayer hybridization between gapped Dirac cones of the two layers.

The Haldane mass term breaks time-reversal symmetry and, therefore, the bands may exhibit nonzero Chern numbers. In undriven TBG, the lower and upper bands cross and, thus, a Chern number cannot be defined for each of them separately. Upon driving the system, a nonzero gap $\delta$ between the lower and the upper bands near $E=0$ is opened. We calculate the Chern number of these bands resulting from the addition of Haldane mass term near the $K$ point as presented in Appendix B. At angles $\theta \geqslant \theta^{*}$, a nonzero Chern number is obtained for weak driving as shown in Fig. 5 for a single spin and valley for $w_{0} / w_{1}=0.8$, which gives $\theta^{*} \approx 1^{\circ}$. The nonzero Chern number at $\theta \geqslant \theta^{*}$ is maintained upon increasing the drive strength until a critical drive strength is reached where the bands cross with the nearest remote bands above and below the upper and lower flat Floquet bands. This crossing occurs since for $\theta \geqslant \theta^{*}$, the gap $\Delta$ decreases as the drive strength is increased from zero [cf. Fig. 3(d) at $\theta=1^{\circ}$ ].
For larger drive strength than this critical value, the Chern numbers are trivial.

Using these Chern numbers, we can obtain the values of the $W_{\varepsilon}^{3}$ topological invariant $[45,46]$ in the spectral gaps adjacent to the flat Floquet bands. The $W_{\varepsilon}^{3}$ topological invariant at a particular quasienergy $\varepsilon$ counts the total number of chiral edge states at that quasienergy. The value of $W_{\varepsilon=0}^{3}$ at the limit of high driving frequencies can be obtained by first considering a TBG in which all interlayer couplings are zero. In this case, the system corresponds to two copies of single-layer graphene. Illumination by circularly polarized light opens a gap between the Dirac nodes and, for each spin, yields two edge states. Importantly, increasing the interlayer tunneling to its physical value does not close the gap $\delta$, implying that the number of edge states in this gap remains the same. Therefore, in the driven TBG system, we study $W_{\varepsilon=0}^{3}=-1$ for a single spin and valley. The $W_{\varepsilon}^{3}$ invariant for any quasienergy $\varepsilon$ is related to the Chern number $C_{\varepsilon, \varepsilon^{\prime}}$ of the band lying between quasienergies $\varepsilon$ and $\varepsilon^{\prime}$, where $\varepsilon>\varepsilon^{\prime}$ and satisfies $W_{\varepsilon}^{3}=W_{\varepsilon^{\prime}}^{3}+C_{\varepsilon, \varepsilon^{\prime}}[45,46]$. Therefore, $W_{\varepsilon}^{3}$ above (below) the upper (lower) flat Floquet bands can be computed using the computed Chern numbers presented in Fig. 5.

\section{LATTICE RELAXATION EFFECTS}

The interplay between the vdW interaction energy and the elastic energy at the interface leads to structural reconstruction of TBG. This reconstruction includes expansion of the $A B / B A$ domains at the expense of the $A A / B B$ domains, periodic variations in the height, and appearance of sharp solitonic boundaries between the different types of domains [47]. These effects introduce additional energy scales, such as the width of the boundary, which become important at small twist angles.

To validate the emergence of optically induced Floquet flat bands at small twist angles, we have extended the generalized continuum model in Refs. $[39,48]$ which reproduces the results of discrete Fourier transform-quality tight-binding Hamiltonians. The extended model, includes the interaction of TBG with a circularly polarized optical driving field. The static Hamiltonian of the generalized model $\tilde{H}$ is given by

$$
\tilde{H}=\left(\begin{array}{cc}
\tilde{h}_{1}(\mathbf{r})+\tilde{A}_{1}(\mathbf{r}) & \tilde{T}(\mathbf{r}) \\
\tilde{T}^{\dagger}(\mathbf{r}) & \tilde{h}_{2}(\mathbf{r})+\tilde{A}_{2}(\mathbf{r})
\end{array}\right),
$$

where $\tilde{h}_{1,2}$ are the low-energy Hamiltonians of the two rotated graphene layers, $\tilde{T}$ is the generalized interlayer coupling term, and $\tilde{A}_{1,2}$ is the in-plane pseudogauge field coupled to the Dirac electron, generated from the geometric deformation and strain for each layer. To account for the formation of the domain line, $\tilde{T}$ includes up to ten orders of the momentum-independent and angle-dependent interlayer tunneling processes of the interlayer coupling in the reciprocal Fourier space as well as additional first-order terms with explicit momentum dependence in the $\mathrm{mBz} . \tilde{A}$ includes first-order angle-dependent intralayer tunneling terms. The numerical values of these terms is adapted from Refs. [39,48] using the parameters of the "full relaxation model." Recall that the interlayer term $T$ of the simple model in Eq. (1) includes only the first-order momentum-independent and angle-independent interlayer process, neglecting the other terms considered here. 

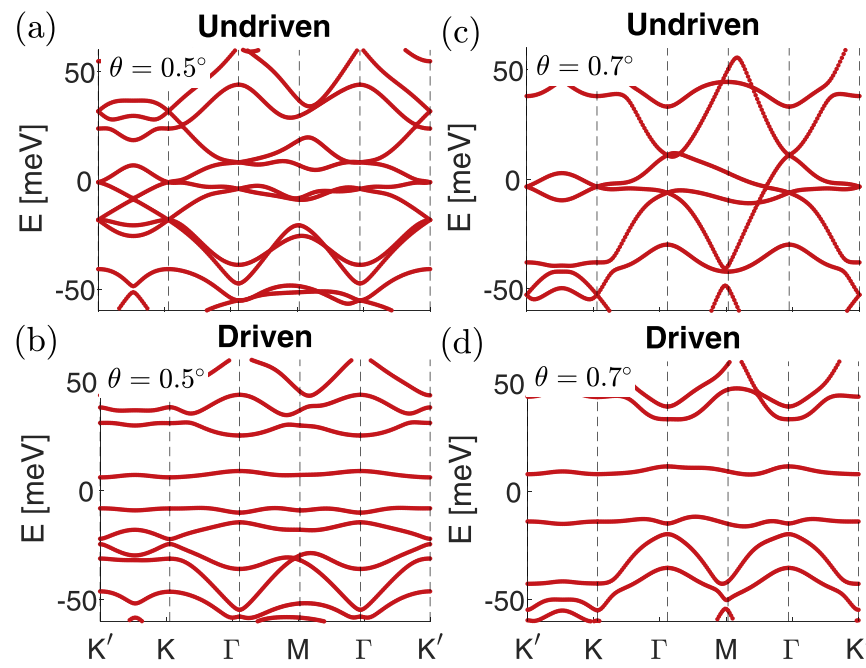

(d)

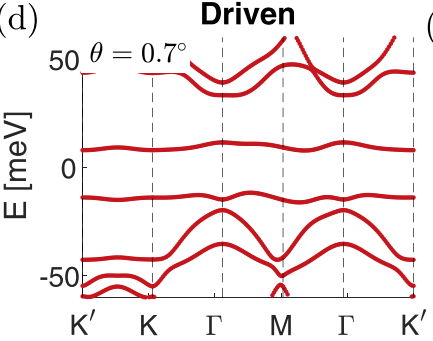

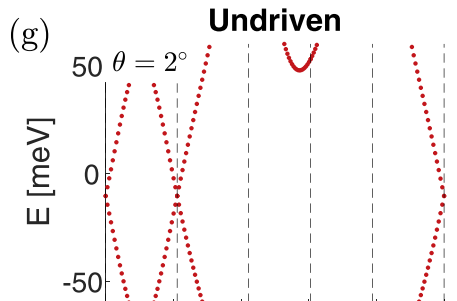
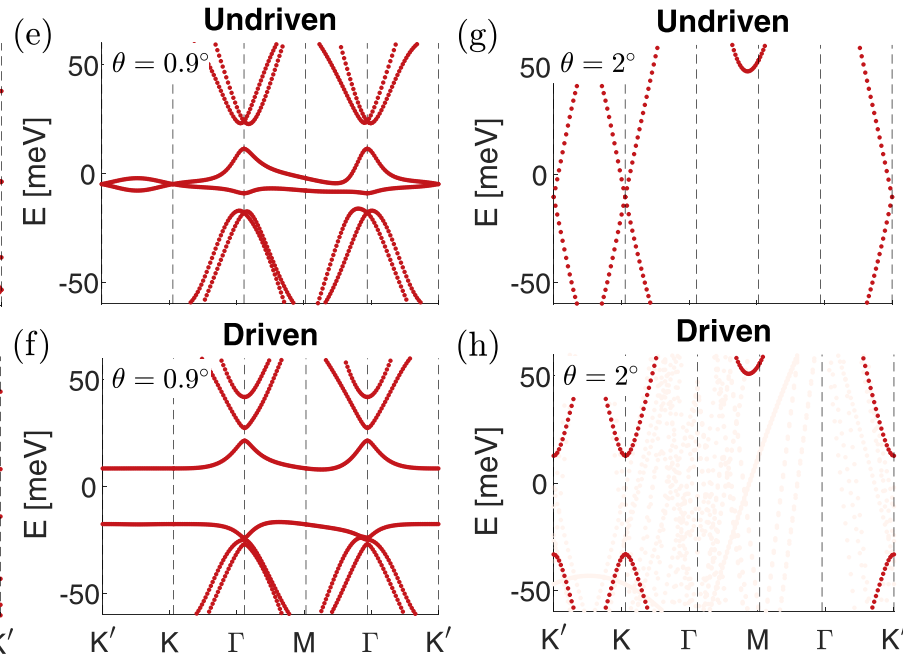

FIG. 6. Floquet band structure of TBG including lattice relaxation effects. (a)-(d) At small twist angles $\left(\theta=0.5^{\circ}\right.$ and $\left.\theta=0.7^{\circ}\right)$, the drive flattens the upper band near $E=0$ and opens a gap from remote bands. (e) and (f) At $\theta=0.9^{\circ}$, a gap $\delta$ between the upper and lower bands near $E=0$ is opened but the gap $\Delta$ from remote bands decreases. (g) and (h) For larger twist angles at $\theta=2^{\circ}$, the driven bands are gapped but are not flattened by the drive. The upper and lower bands near $E=0$ become gapped, resulting with nearly flat bands. In panels (b), (d), (f), and (h), we plot the time-averaged density of states $\bar{\rho}_{0}(E)$ for a driving field of frequency $\hbar \Omega=3 \mathrm{eV}$ and photoinduced gap of $P=63$ meV. Only Floquet states with significant spectral weight $\left[A_{v}^{0}(\mathbf{k})>0.05\right.$, cf. Eq. (3) $]$ are shown.

In Fig. 6, we present the driven and undriven Floquet band structures of the generalized model for various twist angles at $P=63 \mathrm{meV}$ and $\hbar \Omega=3 \mathrm{eV}$. For $\theta=0.5^{\circ}, 0.7^{\circ}$, spectrally isolated flat bands near $E=0$ are induced by the optical driving, properties which are distinct from the band structure of the undriven system as shown in Figs. 6(a)-6(d). The effect of the optical drive on the spectrum of the simple model in Eq. (1) and generalized model Eq. (6) is qualitatively similar: The bands near $E=0$ are flattened, and the gaps $\delta$ and $\Delta$ are opened for any $\theta \leqslant 0.8^{\circ}$. One modification with respect to the spectrum obtained within the simple model, however, is a smaller gap for the lower band near $E=0$ from other remote bands. We associate the emerging asymmetry of the upper and lower bands near $E=0$ with the electronic nature of these bands at small twist angles. The upper band is associated with the electronic orbitals of the domain wall whereas the lower bands are associated with the electronic orbitals of the $A A$ / $B B$ domains [39]. At larger twist angles, $\theta>0.8^{\circ}$, the bandwidth of the driven bands near $E=0$ increases, and the gap to remote bands for the upper band starts to decrease as shown in Figs. 6(e) and 6(f) for $\theta=0.9^{\circ}$. Finally, for angles greater than the magic angle, both interlayer coupling and reconstruction effects have minor effects on the spectrum, which is dominated by the dispersion of the twisted graphene layers. Consequently, for these angles, the Dirac cones at $K$ and $K^{\prime}$ become apparent again as shown in Figs. $6(\mathrm{~g})$ and $6(\mathrm{~h})$ for $\theta=2^{\circ}$. For these angles, the drive mainly opens the gap between the Dirac cones.

The generalized model utilizes numerous parameters to describe the effects of lattice relaxation at any angle. The simple model presented in Sec. II, on the other hand, partially captures the lattice relaxation effects whereas using a single parameter $w_{0} / w_{1}$. For $w_{0}<w_{1}$, this parameter reduces the interlayer coupling in the $A A / B B$ regions with respect to the $A B / B A$ regions. In Appendix $C$, we present the robustness of the results obtained using the simple model to variation of the lattice relaxation parameter $w_{0} / w_{1}$.

\section{DISCUSSION}

In conclusion, we show that driving TBG with UV to near-infrared light can lead to the appearance of flat bands in the Floquet spectrum. The effect persists at a wide range of twist angles, enabling to engineer flat bands without the need of accurate tuning of the relative twist angle between the graphene layers. Thus, Floquet engineering of flat bands may play a particular important role for twisted van der Waals heterostructures which typically exhibit longwavelength nonuniformity in the twist angle.

In this paper, we focused on the Floquet spectrum of twisted bilayer graphene subjected to coherent optical driving field. The Floquet representation is most fruitful in describing the system's properties when the state of electrons at energies near $E \approx 0$ is described by the occupations of the flat Floquet bands, dominating the spectral function $\rho_{0}(\mathbf{k}, E)$ at these energies. The extent to which such a description is useful depends on the timescales and balance of heating and cooling mechanisms in the driven system [35]. Heating mechanism in the driven TBG system mainly arise due to "Floquetumklapp" electron-phonon and electron-electron scattering processes in which the total final quasienergy of the particles differs from the initial one by a quanta of $\hbar \Omega$. Cooling, on the other hand, arises predominantly due to phonon-assisted scattering.

The Floquet-umklapp processes involving the flat Floquet bands near $E \approx 0$ arise predominantly due to off-resonant coupling at driving frequencies above $\hbar \Omega^{*}$ (cf. Sec. IV). As a result, Floquet-umklapp processes involving electrons in these bands are suppressed in powers of $e v \mathcal{E} /\left(\hbar \Omega^{2}\right)$, where $v$ is the Fermi velocity in graphene. The interaction of the drive 
is, however, resonant for bands at energies $E= \pm \hbar \Omega / 2$ at a ring of momenta in the extended Brillouin zone as shown in Fig. 1(b). These resonances cause excitation of electrons into high-energy states which lead to a nonequilibrium carrier distribution (a similar situation occurs in single-layer graphene). At short timescales, the flat bands can be probed using ultrafast techniques as long as the density of excited electrons remains small and the equilibration between the excited electrons and the flat Floquet bands has not yet occurred [28]. At long times, the excited electrons will equilibrate with electrons in the flat bands near $E \approx 0$ and affect their many-body state via various scattering processes [49-53]. Yet, with the introduction of efficient cooling mechanisms, the flat Floquet bands may play an important role in the electronic steady states of irradiated samples [54-58].

The Floquet band structure can be observed via spectroscopic methods, such as time-resolved angle-resolved photoemission spectroscopy [27], and transport properties can be measured via ultrafast electrical measurements [28]. The possibility to drive the material with standard pulsed lasers in the visible-infrared range renders the experimental parameters similar to measurements of single-layer graphene [32].

Note added. After the initial submission of this paper, we became aware of Ref. [59], which proposes the use of UV light to obtain topological bands in low-angle TBG.

\section{ACKNOWLEDGMENTS}

We are grateful to F. von Oppen for helpful comments and to S. Fang for helpful discussions. N.H.L. and O.K. acknowledge financial support from the European Research Council (ERC) under the European Union Horizon 2020 Research and Innovation Programme (Grant Agreement No. 639172). We acknowledge support from the IQIM, an NSF Physics Frontier Center funded by Gordon and Betty Moore Foundation. We are grateful for support from ARO MURI W911NF-16-10361 "Quantum Materials by Design with Electromagnetic Excitation" sponsored by the U.S. Army, as well as NSF grant 1839271. G.R. is also grateful for support from the Simons Foundation and the Packard Foundation. This work was performed, in part, at Aspen Center for Physics, which is supported by National Science Foundation Grant No. PHY1607611.

\section{APPENDIX A: FOURIER REPRESENTATION OF THE HAMILTONIAN}

In this Appendix, we describe the Fourier representation of the Floquet Hamiltonian. The set of modes $\left|\psi_{v}^{(m)}\right\rangle$ used in the main text are the eigenmodes of the Floquet Hamiltonian satisfying the time-independent eigenvalue equation,

$$
\sum_{n=-\infty}^{\infty} \mathcal{H}^{m n}\left|\psi_{v}^{(n)}\right\rangle=\varepsilon_{v}\left|\psi_{v}^{(m)}\right\rangle,
$$

where $\mathcal{H}^{m n}$ represents a block of the infinite Floquet Hamiltonian in the extended zone, given by

$$
\mathcal{H}^{m n}=m \hbar \Omega \delta_{m n}+\frac{\Omega}{2 \pi} \int_{0}^{2 \pi / \Omega)} d t e^{-i(m-n) \Omega t} H(t) .
$$

We start with the representation of the Hamiltonian of the undriven system.

\section{Static Hamiltonian}

We use a convention where the top layer is rotated by an angle $\theta / 2$ and the bottom layer is rotated by an angle $-\theta / 2$. We generalize the representation in Refs. $[9,60]$ and represent the Hamiltonian of the first monolayer of graphene rotated by an angle $\theta / 2$ in the reciprocal space with

$$
h_{1}\left(\theta / 2, \mathbf{k}^{\prime}\right)=-\tau f\left(\theta / 2, \mathbf{k}^{\prime}\right) \sigma_{1+}+\text { H.c., }
$$

where $\sigma_{1+}=\left|\mathbf{k}^{\prime}, 1 A\right\rangle\left\langle\mathbf{k}^{\prime}, 1 B\right|$ is the Pauli matrix using the isospin basis, 1 denotes the top layer (whereas 2 denotes the bottom layer) and $f\left(\theta / 2, \mathbf{k}^{\prime}\right)=\sum_{i=1}^{3} e^{i \mathbf{k}^{\prime} \delta_{i}^{\prime}}$ is the graphene dispersion relation with $\delta_{1}=(0, a)$ and $\delta_{2,3}=a(\mp \sqrt{3}, 1) / 2$. Here, and throughout the paper, primed variables correspond to the top (1), e.g., $\mathbf{k}^{\prime}=R_{z}(\theta / 2) \mathbf{k}$ and $\delta_{1}^{\prime}=R_{z}(\theta / 2) \delta_{1}$ whereas $R_{z}(\theta)$ is the rotation matrix around the $z$ direction which is normal to the plane of the sample. Similarly, the Hamiltonian of the second layer is given by

$$
h_{2}\left(-\theta / 2, \mathbf{k}^{\prime \prime}\right)=-\tau f\left(-\theta / 2, \mathbf{k}^{\prime \prime}\right) \sigma_{2+}+\text { H.c., }
$$

where $\sigma_{2+}=\left|\mathbf{k}^{\prime \prime}, 2 A\right\rangle\left\langle\mathbf{k}^{\prime \prime}, 2 B\right|$ and $\mathbf{k}^{\prime \prime}=R_{z}(-\theta / 2) \mathbf{k}$ and $f\left(-\theta / 2, \mathbf{k}^{\prime \prime}\right)=\sum_{i=1}^{3} e^{i \mathbf{k}^{\prime \prime} \delta_{i}^{\prime \prime}}$.

The interlayer interaction operator $T(\mathbf{k})$ can be represented by an infinite matrix using the plane-wave expansion with elements $T_{\mathbf{k}^{\prime}, \mathbf{p}^{\prime \prime}}^{\alpha \beta} \equiv\left\langle\mathbf{k}^{\prime}, 1 \alpha\left|H_{\perp}\right| \mathbf{p}^{\prime \prime}, 2 \beta\right\rangle$. The superscripts $\alpha, \beta \in$ $\{A, B\}$ denote the isospin of the monolayer and the indices 1 and 2 indicate the top and bottom layers, respectively. Note that $\mathbf{k}^{\prime}$ and $\mathbf{p}^{\prime \prime}$ are defined in the repeated reciprocal zone and measured from the center of the Brillouin zone (and not relative to the Dirac point). The interlayer interaction Hamiltonian $H_{\perp}$ can be represented using a tight binding in real space,

$$
H_{\perp}=\sum_{i j} t_{\perp i j} \hat{c}_{1 i}^{\dagger} \hat{c}_{2 j}+\text { H.c. },
$$

where $\hat{c}_{1 i}$ and $\hat{c}_{2 j}$ are the fermionic annihilation operators of the top and bottom layers, respectively, the indices $i, j$ denote the real-space lattice point, and $t_{\perp i j}$ denote the interlayer hopping parameters. We invoke the two-center approximation, assuming that the hopping parameters depend only on the relative distance between the different lattice points $t_{\perp i j}=$ $t_{\perp}\left(\left|R_{i}-R_{j}\right|\right)$ where $R_{i}$ denotes the position of the $i$ th site. Representing $H_{\perp}$ in the reciprocal Fourier space yields the interlayer coupling coefficients $[9,60]$,

$$
\begin{aligned}
T_{\mathbf{k}^{\prime}, \mathbf{p}^{\prime \prime}}^{\alpha \beta}= & \sum_{\mathbf{G}_{1}^{\prime}, \mathbf{G}_{2}^{\prime}} \frac{t_{\perp}\left(\mathbf{k}^{\prime}+\mathbf{G}_{1}^{\prime}\right)}{A_{\mathrm{uc}}} e^{i\left[\mathbf{G}_{1}^{\prime} \delta_{\alpha}^{\prime}-\mathbf{G}_{2}^{\prime}\left(\delta_{\beta}^{\prime}-a\right)-R_{z}(-\theta) \mathbf{G}_{2}^{\prime} \mathbf{d}\right]} \\
& \times \delta_{\mathbf{k}^{\prime}+\mathbf{G}_{1}^{\prime}, \mathbf{p}^{\prime \prime}+R_{z}(-\theta) \mathbf{G}_{2}^{\prime} .}
\end{aligned}
$$

Here, we sum over the reciprocal lattice vectors $\mathbf{G}_{1}^{\prime}$ and $\mathbf{G}_{2}^{\prime}$ of the two layers, $A_{\mathrm{uc}}$ is the unit-cell area and $d$ is the spacing between the two layers. The parameters $\boldsymbol{\delta}_{\alpha}^{\prime}$ and $\boldsymbol{\delta}_{\boldsymbol{\beta}}{ }^{\prime}$ indicate the relative position of the $A$ and $B$ atoms within a unit cell in the top layer with $\boldsymbol{\delta}_{A}^{\prime}=\mathbf{0}$ and $\boldsymbol{\delta}_{B}^{\prime}=(0, a)$. The Kronecker- $\delta$ $\delta_{\mathbf{k}^{\prime}+\mathbf{G}_{1}^{\prime}, \mathbf{p}^{\prime \prime}+R_{z}(-\theta) \mathbf{G}_{2}^{\prime}}$ ensures crystal momentum conservation by the tunneling process. To simplify the expression of $T_{\mathbf{k}^{\prime}, \mathbf{p}^{\prime \prime}}^{\alpha \beta}$ further, it is plausible to assume that the interlayer tunneling is slowly varying in space due to the large interlayer separation 
$d>a$ such that $t_{\perp}(\mathbf{k})$ falls rapidly to zero at high momenta (cf. Refs. [9,60]). We can, then, truncate the sum over all reciprocal lattice vectors to a sum over the three smallest reciprocal lattice vectors (which are of the same magnitude) yielding

$$
T_{\mathbf{k}^{\prime}, \mathbf{p}^{\prime \prime}}^{\alpha \beta} \approx \sum_{i=1}^{3} \delta_{\mathbf{k}^{\prime}+\mathbf{q}_{i}, \mathbf{p}^{\prime \prime}} T_{i}^{\alpha \beta}
$$

where the momenta $\mathbf{q}_{i}$ are given in the main text. The $2 \times 2$ matrices $T_{i}$ in the isospin basis which are given in the main text include lattice relaxation effects. Equation (A7) can be

$$
H_{k}^{(1)}=\left(\begin{array}{cc}
h_{1}\left(\theta / 2, \mathbf{k}^{\prime}\right) & T_{1} \\
T_{1}^{\dagger} & h_{2}\left(-\theta / 2, \mathbf{k}^{\prime}+\mathbf{q}_{1}\right) \\
T_{2}^{\dagger} & \\
T_{3}^{\dagger} &
\end{array}\right.
$$

A diagrammatic representation of the Hamiltonian in the reciprocal space is shown in Fig. 7 for $r_{\max }=13$. In this representation, the central node has a momentum k. Each node represents a $2 \times 2$ Hamiltonian of monolayer graphene, whereas black nodes represent the matrices $h_{1}\left(\theta / 2, \mathbf{k}^{\prime}+\mathbf{k}_{n}\right)$ whereas the gold-colored nodes represent $h_{2}\left(-\theta / 2, \mathbf{k}^{\prime}+\mathbf{k}_{n}\right)$. Here, $\mathbf{k}_{n} \equiv \mathbf{k}_{l m p}=l \mathbf{g}_{1}+m \mathbf{g}_{2}+p \mathbf{q}_{1}$ denotes the hexagon reciprocal grid in the extended zone. This grid is defined by the moiré reciprocal lattice vectors $\mathbf{g}_{1}=\mathbf{q}_{1}-\mathbf{q}_{3}$, and $\mathbf{g}_{2}=$ $\mathbf{q}_{2}-\mathbf{q}_{1}$, the integers $l, m$, and the basis index $p= \pm 1$. Note that, in the main text, we denote the different combinations of $l, m$, and $p$ with a single index $n$ for brevity. A nonzero transition matrix connecting layers 1 to 2 is represented by an edge where the transition matrix $T_{1}$ is indicated by a blue

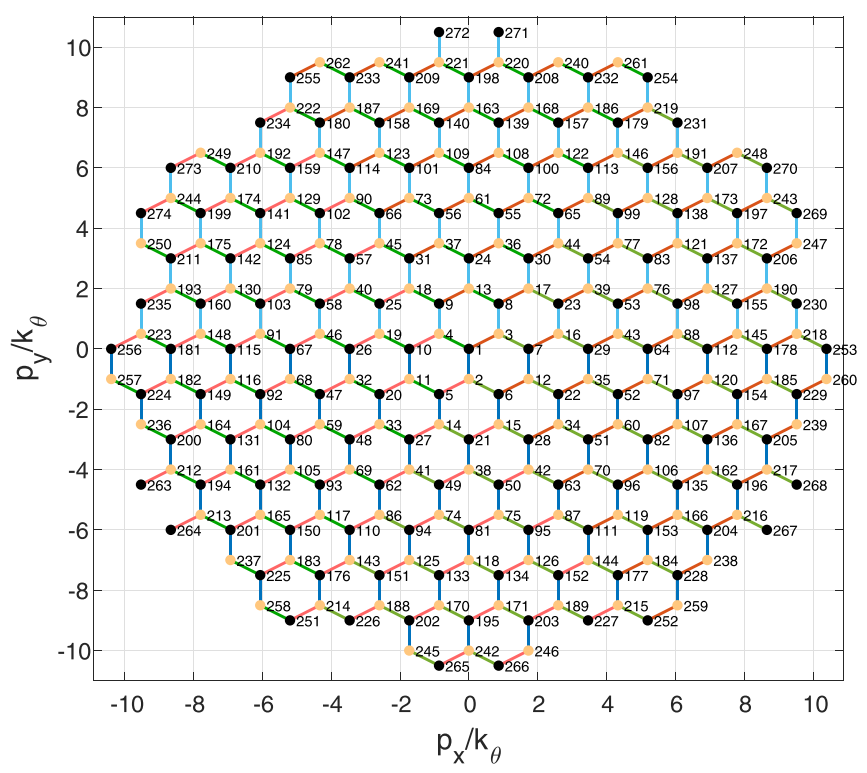

FIG. 7. Reciprocal basis representation of the static Hamiltonian $H(\mathbf{k})$. The nodes represent the monolayer graphene Hamiltonians whereas the edge describes the interlayer couplings. This representation is truncated with a maximal hopping number $r_{\max }=13$. considered as a nearest-neighbors hopping Hamiltonian in the reciprocal moiré lattice.

The expansion of $T_{\mathbf{k}^{\prime}, \mathbf{p}^{\prime \prime}}^{\alpha \beta}$ couples the wave-function $\left|\psi_{1}\left(\mathbf{k}^{\prime}\right)\right\rangle$ in the first layer with the wave functions of the other layer $\left|\psi_{2}\left(\mathbf{p}^{\prime \prime}\right)\right\rangle$ for $\mathbf{p}^{\prime \prime}=\mathbf{k}^{\prime}+\mathbf{q}_{1}, \mathbf{p}^{\prime \prime}=\mathbf{k}^{\prime}+\mathbf{q}_{2}$, and $\mathbf{p}^{\prime \prime}=\mathbf{k}^{\prime}+\mathbf{q}_{3}$. In general, this matrix couples points on a hexagonal lattice in reciprocal space connected by reciprocal lattice vectors of the moiré lattice. To numerically solve this infinite expansion, we pose a further (numerical) truncation by setting a maximal hopping number $r_{\max }$, where the total number of sites considered is $\frac{3}{2} r_{\max }^{2}+\frac{3}{2} r_{\max }+1$. As an example, the Hamiltonian for $r_{\max }=1$ is given by

$$
\left.\begin{array}{cc}
T_{2} & T_{3} \\
h_{2}\left(-\theta / 2, \mathbf{k}^{\prime}+\mathbf{q}_{2}\right) & \\
& h_{2}\left(-\theta / 2, \mathbf{k}^{\prime}+\mathbf{q}_{3}\right)
\end{array}\right) .
$$

edge, a transition matrix $T_{2}$ is indicated by a red edge, and a transition matrix $T_{3}$ is indicated by a green edge. Note that the coupling between layers 2 to 1 is represented in a similar way but with the Hermitian conjugated matrices $T_{1}^{\dagger}, T_{2}^{\dagger}$, and $T_{3}^{\dagger}$. The numbers near each point indicate the index of the vector basis in the numerical matrix (not including the isospin degree of freedom). It is useful to write the general static Hamiltonian in reciprocal space with

$$
H^{\left(r_{\max }\right)}(\mathbf{k})=T(\mathbf{k})+\sum_{p=0}^{1} \sum_{l, m}|l, m, p\rangle h_{l m p}(\theta / 2, \mathbf{k})\langle l, m, p|,
$$

where the sum is over all integers $l, m$ satisfying $r(l, m, p)=$ $|l||+| m|+| l+m-p \mid \leqslant r_{\max }$. The hopping number $r$ counts the number of edges connecting that $\mathbf{k}_{l m p}$ grid point to the momentum $\mathbf{k}$. For even values of $r(l, m, p)$, we identify $h_{l m p}=h_{1}\left(\theta / 2, \mathbf{k}^{\prime}+\mathbf{k}_{l m p}\right)$ whereas for odd values of $r(l, m, p)$, we have $h_{l m p}=h_{2}\left(-\theta / 2, \mathbf{k}^{\prime}+\mathbf{k}_{l m p}\right)$. In general, $r_{\max }$ is determined upon convergence of the result of the low energy (we verify that the error is less than $1 \%$ in the low-energy spectrum of $|E| \leqslant 50 \mathrm{meV}$ ), and we typically use $13 \leqslant r_{\max } \leqslant 22$ in our calculations.

\section{Floquet Hamiltonian}

We construct the time-dependent driven Hamiltonian by applying the Peierls substitution in reciprocal space $\mathbf{k} \rightarrow \mathbf{k}-$ $e \mathbf{A}(t) / \hbar$, where $\mathbf{A}=\mathcal{E} / \Omega$ is the vector potential. Although the interlayer interaction is independent of the normally incident drive field, the monolayer Hamiltonian is transformed via $h_{1}\left(\theta / 2, \mathbf{k}^{\prime}\right) \rightarrow h_{1}\left[\theta / 2, \mathbf{k}^{\prime}-e \mathbf{A}^{\prime}(t) / \hbar\right]$ for the first layer and $h_{2}\left(-\theta / 2, \mathbf{k}^{\prime \prime}\right) \rightarrow h_{2}\left[-\theta / 2, \mathbf{k}^{\prime \prime}-e \mathbf{A}^{\prime \prime}(t) / \hbar\right]$ for the second layer. We expand the Hamiltonian in a harmonic expansion,

$$
\begin{aligned}
h_{1}\left[\theta / 2, \mathbf{k}^{\prime}-e a \mathbf{A}^{\prime}(t) / \hbar\right]= & -\tau \sum_{n=-\infty}^{\infty}\left[g_{+}\left(\theta / 2, \mathbf{k}^{\prime}, n\right) \sigma_{1+}\right. \\
& \left.+g_{-}\left(\theta / 2, \mathbf{k}^{\prime}, n\right) \sigma_{1-}\right] e^{i n \Omega t}
\end{aligned}
$$


where

$$
\begin{aligned}
g_{ \pm}\left(\theta / 2, \mathbf{k}^{\prime}, n\right)= & i^{n} e^{i n(\phi+\theta / 2)}\left[e^{ \pm i \mathbf{k}^{\prime} \delta_{1}^{\prime}} J_{n}\left(\mp \frac{e a}{\hbar} A\right)+e^{ \pm i \mathbf{k}^{\prime} \delta_{2}^{\prime}}\right. \\
& \left.\times e^{-i n \psi_{+}} J_{n}\left( \pm \Gamma_{+}\right)+e^{ \pm i \mathbf{k}^{\prime} \delta_{3}^{\prime}} e^{-i n \psi_{-}} J_{n}\left( \pm \Gamma_{-}\right)\right]
\end{aligned}
$$

are the coefficients of the $n$th harmonic order for the first layer. The parameters $\Gamma \pm$ and $\psi_{ \pm}$will be defined below. Similarly, for the second layer, we use

$$
\begin{aligned}
h_{2}\left(-\theta / 2, \mathbf{k}^{\prime \prime}-e a \mathbf{A}^{\prime \prime}(t) / \hbar\right) \\
=-\tau \sum_{n=-\infty}^{\infty}\left[g_{+}\left(-\theta / 2, \mathbf{k}^{\prime \prime}, n\right)\right. \\
\left.\quad \times \sigma_{2+}+g_{-}\left(-\theta / 2, \mathbf{k}^{\prime \prime}, n\right) \sigma_{2-}\right] e^{i n \Omega t},
\end{aligned}
$$

where the coefficients of the $n$th harmonic order of the second layer are given by

$$
\begin{aligned}
g_{ \pm}\left(-\theta / 2, \mathbf{k}^{\prime \prime}, n\right)= & i^{n} e^{i n(\phi-\theta / 2)}\left[e^{ \pm i \mathbf{k}^{\prime \prime} \delta_{1}^{\prime \prime}} J_{n}\left(\mp \frac{e a}{\hbar} A\right)\right. \\
& +e^{ \pm i \mathbf{k}^{\prime \prime} \delta_{2}^{\prime \prime}} e^{-i n \psi_{+}} J_{n}\left( \pm \Gamma_{+}\right) \\
& \left.+e^{ \pm i \mathbf{k}^{\prime \prime} \delta_{3}^{\prime \prime}} e^{-i n \psi_{-}} J_{n}\left( \pm \Gamma_{-}\right)\right]
\end{aligned}
$$

The above relations are derived using the identities [61],

$$
\begin{gathered}
e^{i z \cos \phi}=\sum_{n=-\infty}^{\infty} i^{n} J_{n}(z) e^{i n \phi} \\
e^{-i q \psi} J_{q}(\Gamma)=\sum_{n=-\infty}^{\infty} J_{n+q}(\alpha) J_{n}(\beta) e^{-i \phi n}
\end{gathered}
$$

where $J_{n}(z)$ is the $n$ th-order Bessel function and $\phi$ is the phase retardance between $A_{x}$ and $A_{y}$. The parameters $\Gamma$ and $\psi$ in Eqs. (A14) and (A15) can be written by two parameters $\alpha$ and $\beta$ as

$$
\begin{gathered}
\Gamma=\sqrt{\alpha^{2}+\beta^{2}-2 \alpha \beta \cos (\phi)}, \\
\psi=\operatorname{atan}\left(\frac{\beta \sin (\phi)}{\alpha-\beta \cos (\phi)}\right) .
\end{gathered}
$$

In our case, we find that $\Gamma_{ \pm}$and $\psi_{ \pm}$in Eqs. (A10)-(A12) are given by

$$
\Gamma_{ \pm}=\frac{a}{2} \sqrt{A_{y}^{2}+3 A_{x}^{2} \pm 2 \sqrt{3} A_{x} A_{y} \cos (\phi)},
$$

and

$$
\psi_{ \pm}=\operatorname{atan}\left(\frac{\sqrt{3} A_{x} \sin (\phi)}{ \pm A_{y}+\sqrt{3} A_{x} \cos (\phi)}\right) .
$$

For thespecial case of circularly polarized light considered here, we use $\phi=\pi / 2$ and obtain the simple relations $\Gamma_{ \pm}=e a A / \hbar$ and $\psi_{ \pm}= \pm \pi / 3$. We can use the expansion in Eq. (A10) with a truncated sum on integers from $-N_{F}$ to $N_{F}$ in order to construct the time-independent Floquet Hamiltonian
$\mathcal{H}_{m n}$ with $-N_{\mathrm{F}} \leqslant m \leqslant N_{\mathrm{F}}$, yielding

$$
\begin{aligned}
\mathcal{H}_{m, m+n}(\mathbf{k})= & {\left[m \Omega \sigma_{0}+T(\mathbf{k})\right] \delta_{m n}+\sum_{p^{\prime}=0}^{1} \sum_{l^{\prime}, m^{\prime}}\left|l^{\prime}, m^{\prime}, p^{\prime}\right\rangle } \\
& \times \tilde{h}_{l^{\prime} m^{\prime} p^{\prime}}(\theta / 2, \mathbf{k}, n)\left\langle l^{\prime}, m^{\prime}, p^{\prime}\right|,
\end{aligned}
$$

where, for even values of $r\left(l^{\prime}, m^{\prime}, p^{\prime}\right)$, we define

$$
\begin{aligned}
\tilde{h}_{l^{\prime} m^{\prime} p^{\prime}}\left(\theta / 2, \mathbf{k}^{\prime}, n\right)= & -\tau\left[g_{+}\left(\theta / 2, \mathbf{k}^{\prime}+\mathbf{k}_{l^{\prime} m^{\prime} p^{\prime}}, n\right) \sigma_{1+}\right. \\
& \left.+g_{-}\left(\theta / 2, \mathbf{k}^{\prime}+\mathbf{k}_{l^{\prime} m^{\prime} p^{\prime}}, n\right) \sigma_{1-}\right],
\end{aligned}
$$

and, for odd values of $r\left(l^{\prime}, m^{\prime}, p^{\prime}\right)$, we define

$$
\begin{aligned}
\tilde{h}_{l^{\prime} m^{\prime} p^{\prime}}\left(\theta / 2, \mathbf{k}^{\prime}, n\right)= & -\tau\left[g_{+}\left(-\theta / 2, \mathbf{k}^{\prime}+\mathbf{k}_{l^{\prime} m^{\prime} p^{\prime}}, n\right) \sigma_{2+}\right. \\
& \left.+g_{-}\left(-\theta / 2, \mathbf{k}^{\prime}+\mathbf{k}_{l^{\prime} m^{\prime} p^{\prime}}, n\right) \sigma_{2-}\right] .
\end{aligned}
$$

In practice, we observe convergence of the numerical calculations when setting a cutoff of $N_{\mathrm{F}}=2$ or 3 . The dimension of the Floquet matrix is, then, $\left(2 N_{\mathrm{F}}+1\right)\left(3 r_{\max }^{2}+3 r_{\max }+2\right)$.

The function $g_{ \pm}( \pm \theta / 2, \mathbf{k}, n)$ obtains a simple form in the low power regime $e a A \ll \hbar$. In this regime, the zeroth order describes the undriven Hamiltonian with $g_{+}( \pm \theta / 2, \mathbf{k}, 0)=$ $f( \pm \theta / 2, \mathbf{k})$ and $g_{-}( \pm \theta / 2, \mathbf{k}, 0)=f^{*}( \pm \theta / 2, \mathbf{k})$. Furthermore, near the $K$ point in the top layer, we find that $g_{ \pm}(\theta / 2, \mathbf{k},-1)=0$ and $g_{ \pm}(\theta / 2, \mathbf{k}, 1) \approx 3 e a A e^{i \theta / 2} /(2 \hbar)$, and near the $K$ Dirac point of the bottom layer, we have $g_{ \pm}(-\theta / 2, \mathbf{k},-1)=0$ and $g_{ \pm}(\theta / 2, \mathbf{k}, 1) \approx 3 e a A e^{-i \theta / 2} /(2 \hbar)$. We also find that the contribution of terms with higher orders of $n$ are smaller by powers of $(e a A / \hbar)^{n}$. Importantly, for $\theta \ll 1$, we find that the effective Hamiltonian in leading powers of the small parameter $x=\left[(3 \tau e a A) /\left(2 \hbar^{2} \Omega\right)\right]$ at these conditions is given by

$$
H_{\text {eff }} \approx H+\left[\mathcal{H}_{01}, \mathcal{H}_{10}\right] /(\hbar \Omega) .
$$

\section{APPENDIX B: TOPOLOGY OF THE FLAT BANDS}

We calculate the Chern number of the upper an lower bands near $E=0$ resulting from the addition of the Haldane mass term near the $K$ point, by following the procedure presented in Refs. $[62,63]$. We discretize the reciprocal space using a rectangular grid $\mathbf{k}=k_{\theta}(m \hat{x}+n \hat{y}) / N_{k}$ for integers $-N_{k} \leqslant$ $m, n \leqslant N_{k}$ and, typically, use $N_{k}=200$. We consider only the grid points within the first $\mathrm{mBz}$ hexagon. Our goal is to diagonalize the static Hamiltonian in Eq. (A9) including a Haldane photoinduced mass term of size $P$.

Recall that the static Hamiltonian Eq. (A9) is valley and spin degenerate. The two valley degenerate bands arise from Bloch wave functions which are superpositions of momenta near the $K$ or $K^{\prime}$ points of both layers. Here, we calculate the Bloch wave functions in the $\mathrm{mBz}$ corresponding to the bands whose momenta $\mathbf{k}$ are always near the $\mathbf{K}$ points of both layers and far from the $\mathbf{K}^{\prime}$ points of both layers since $r_{\max } k_{\theta} \ll \mid \mathbf{K}-$ $\mathbf{K}^{\prime} \mid$. Therefore, to take the Haldane mass term into account, it is sufficient to add to the Hamiltonian $h(k)$ appearing in Eq. (A9) a mass term $P \sigma_{z}$, which represents the Haldane mass term correctly near the $K$ point.

We diagonalize the Hamiltonian in Eq. (A9) including the term $P \sigma_{z} \otimes \sigma_{1}$ and find the wave-functions $\left|\psi_{l}(\mathbf{k})\right\rangle$ of the lower and upper bands denoted with $l \in\{v, c\}$. We, then, 

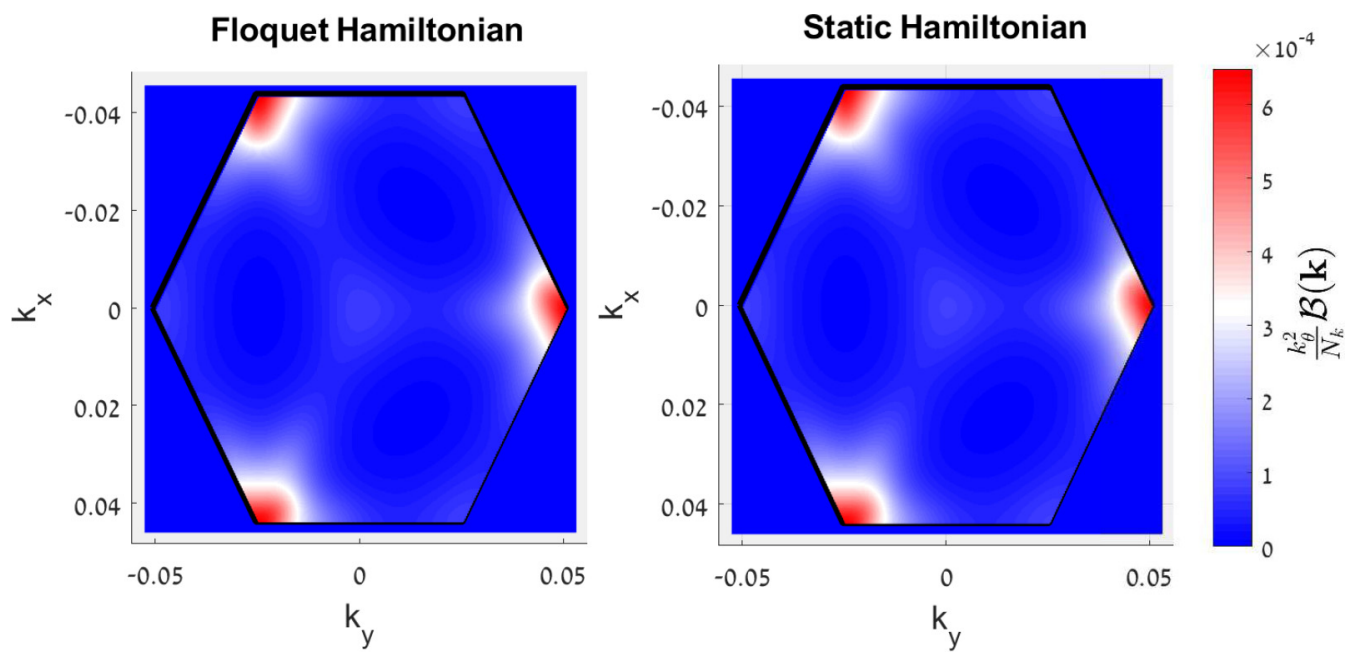

FIG. 8. Berry curvature of the upper band in the $\mathrm{mBz}$ for $\theta=1.2^{\circ}$ and $P=10 \mathrm{meV}$. The effective static Hamiltonian and the full Floquet analysis yield the same result.

calculate the local Berry curvature with

$$
\begin{aligned}
\mathcal{B}_{l}(\mathbf{k})= & \left(\frac{N_{k}}{k_{\theta}}\right)^{2} \arg \left[U_{l, x}(\mathbf{k}) U_{l, y}\left(\mathbf{k}+\hat{x} \frac{k_{\theta}}{N_{k}}\right)\right. \\
& \left.\times U_{l, x}^{*}\left(\mathbf{k}+\hat{y} \frac{k_{\theta}}{N_{k}}\right) U_{l, y}^{*}(\mathbf{k})\right],
\end{aligned}
$$

where the potentials $U_{l, x}(\mathbf{k})$ and $U_{l, y}(\mathbf{k})$ are given by

$$
U_{x}^{v, c}(\mathbf{k})=\left\langle\psi_{v, c}\left(\mathbf{k}+\hat{x} k_{\theta} / N_{k}\right) \mid \psi_{v, c}(\mathbf{k})\right\rangle .
$$

We calculate the Chern number of the lower and upper bands in Fig. 5 in the main text by summing the local Berry curvature over the $\mathrm{mBz}$,

$$
C_{l}=\frac{1}{2 \pi}\left(\frac{k_{\theta}}{N_{k}}\right)^{2} \sum_{\mathbf{k}} \mathcal{B}_{l}(\mathbf{k}) .
$$

An example of the Berry curvature of the upper band of TBG with $\theta=1.2^{\circ}$ driven by $P=10 \mathrm{meV}$ is shown in Fig. 8 for the static Hamiltonian.

At relatively high drive frequencies as shown in the main text, the Floquet bands closely resemble the bands of the static Hamiltonian as mixing and level crossings are suppressed. Therefore, we expect that the Berry curvature of the Floquet bands should closely resemble the Berry curvature of the static calculation with the Haldane mass term up to isolated level crossings which effect the Berry curvature in very narrow regions in the reciprocal space. To demonstrate this, we calculate the Berry curvature of the Floquet bands with the full time-dependent Hamiltonian with $\hbar \Omega=3 \mathrm{eV}$ in Fig. 8 . To perform this calculation, we chose the wave functions of the upper and lower bands as the two wave functions whose spectral weight $A_{v}^{0}(\mathbf{k})$ in the energy interval $|E|<\Delta E$ is maximal, cf. Eq. (3) in the main text [at high drive frequencies, these bands have $\left.A_{v}^{0}(\mathbf{k}) \approx 1\right]$. This example demonstrates that, indeed, the Berry curvature of the static Hamiltonian captures the one of the Floquet bands at relatively high drive frequencies.

\section{APPENDIX C: LATTICE RELAXATION IN THE SIMPLE MODEL}

Lattice relaxation effects lead to various structural deformations of TBG. To leading order, we capture the an expansion of the $A B / B A$ domains at the expense of the $A A / B B$ domains using a single relaxation parameter $w_{0} / w_{1}$. In Fig. 9, we calculate the dependence of the bandwidth and the gaps $\Delta, \delta$ on the parameter $w_{0} / w_{1}$ for $\theta=0.7^{\circ}, 0.9^{\circ}$ using $\hbar \Omega=1.5 \mathrm{eV}$ and $\mathcal{E}=4 \mathrm{MV} / \mathrm{cm}(P=33 \mathrm{meV})$. For undriven TBG, the gap $\delta$ vanishes at the $K$ point for any value of $w_{0} / w_{1}$, and the gap $\Delta$ vanishes at the $\Gamma$ point within the
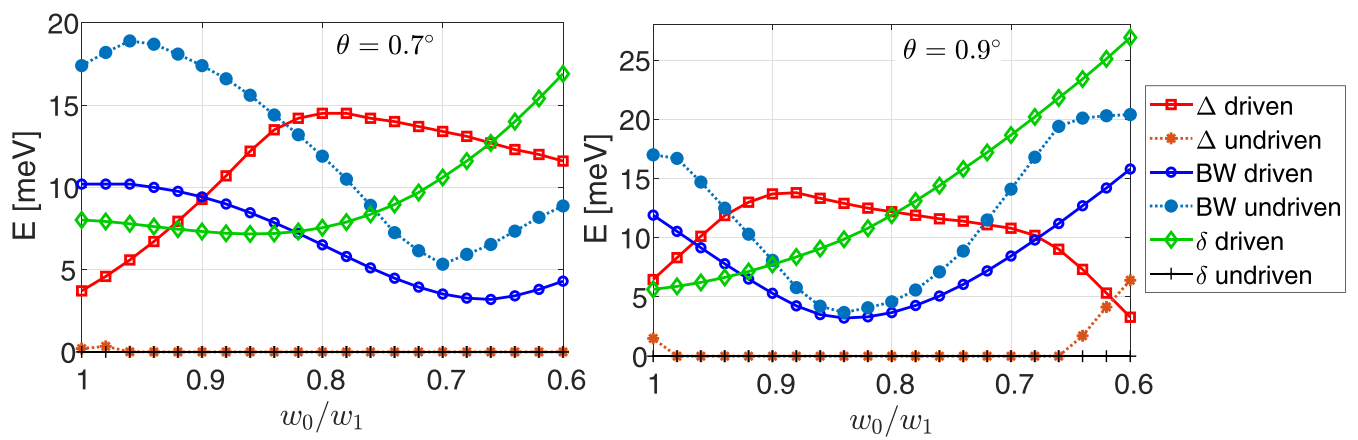

FIG. 9. The increased gaps $\Delta, \delta$, and low bandwidth of driven TBG are robust with variations of lattice relaxation effects. Calculation for $\theta=0.7^{\circ}$ (left) and $\theta=0.9^{\circ}$ (right) using $\hbar \Omega=1.5 \mathrm{eV}$ and $\mathcal{E}=4 \mathrm{MV} / \mathrm{cm}$. 
realistic parameter range of $0.7 \leqslant w_{0} / w_{1} \leqslant 0.85$. The driving field $(P=33 \mathrm{meV}$ and $\hbar \Omega=1.5 \mathrm{eV})$ opens both gaps for a wide range of $w_{0} / w_{1}$. The bandwidth is also reduced by the drive in the same range of lattice relaxation parameters.
Similar results are obtained for other twist angles below the magic angle. We, therefore, conclude that the features induced by the drive are robust to variations in the lattice relaxation parameter of the single model.
[1] A. K. Geim and I. V. Grigorieva, Van der Waals heterostructures, Nature (London) 499, 419 (2013).

[2] K. S. Novoselov, A. Mishchenko, A. Carvalho, and A. H. C. Neto, 2D materials and van der Waals heterostructures, Science 353, aac9439 (2016).

[3] Y. Liu, N. O. Weiss, X. Duan, H.-C. Cheng, Y. Huang, and X. Duan, Van der Waals heterostructures and devices, Nat. Rev. Mater. 1, 16042 (2016).

[4] A. H. MacDonald, Trend: Bilayer graphene's wicked, twisted road, Physics 12, 12 (2019).

[5] J. M. B. Lopes dos Santos, N. M. R. Peres, and A. H. Castro Neto, Graphene Bilayer with a Twist: Electronic Structure, Phys. Rev. Lett. 99, 256802 (2007).

[6] R. Ribeiro-Palau, C. Zhang, K. Watanabe, T. Taniguchi, J. Hone, and C. R. Dean, Twistable electronics with dynamically rotatable heterostructures, Science 361, 690 (2018)

[7] S. Carr, D. Massatt, S. Fang, P. Cazeaux, M. Luskin, and E. Kaxiras, Twistronics: Manipulating the electronic properties of two-dimensional layered structures through their twist angle, Phys. Rev. B 95, 075420 (2017).

[8] J. M. B. Lopes dos Santos, N. M. R. Peres, and A. H. Castro Neto, Continuum model of the twisted graphene bilayer, Phys. Rev. B 86, 155449 (2012).

[9] R. Bistritzer and A. H. MacDonald, Moire bands in twisted double-layer graphene, Proc. Natl. Acad. Sci. USA 108, 12233 (2011).

[10] G. Tarnopolsky, A. J. Kruchkov, and A. Vishwanath, Origin of Magic Angles in Twisted Bilayer Graphene, Phys. Rev. Lett. 122, 106405 (2019).

[11] L. Shi, J. Ma, and J. C. W. Song, Gate-tunable flat bands in van der Waals patterned dielectric superlattices, 2D Mater. 7, 015028 (2019).

[12] Y. Cao, V. Fatemi, S. Fang, K. Watanabe, T. Taniguchi, E. Kaxiras, and P. Jarillo-Herrero, Unconventional superconductivity in magic-angle graphene superlattices, Nature (London) 556, 43 (2018).

[13] Y. Cao, V. Fatemi, A. Demir, S. Fang, S. L. Tomarken, J. Y. Luo, J. D. Sanchez-Yamagishi, K. Watanabe, T. Taniguchi, E. Kaxiras, R. C. Ashoori, and P. Jarillo-Herrero, Correlated insulator behavior at half-filling in magic-angle graphene superlattices, Nature (London) 556, 80 (2018).

[14] K. Kim, A. DaSilva, S. Huang, B. Fallahazad, S. Larentis, T. Taniguchi, K. Watanabe, B. J. LeRoy, A. H. MacDonald, and E. Tutuc, Tunable moire bands and strong correlations in smalltwist-angle bilayer graphene, Proc. Natl. Acad. Sci. USA 114, 3364 (2017).

[15] M. Yankowitz, S. Chen, H. Polshyn, Y. Zhang, K. Watanabe, T. Taniguchi, D. Graf, A. F. Young, and C. R. Dean, Tuning superconductivity in twisted bilayer graphene, Science 363, 1059 (2019).

[16] A. L. Sharpe, E. J. Fox, A. W. Barnard, J. Finney, K. Watanabe, T. Taniguchi, M. A. Kastner, and D. Goldhaber-
Gordon, Emergent ferromagnetism near three-quarters filling in twisted bilayer graphene, Science 365, 605 (2019).

[17] B. Lian, Z. Wang, and B. A. Bernevig, Twisted Bilayer Graphene: A Phonon-Driven Superconductor, Phys. Rev. Lett. 122, 257002 (2019).

[18] N. N. T. Nam and M. Koshino, Lattice relaxation and energy band modulation in twisted bilayer graphene, Phys. Rev. B 96, 075311 (2017).

[19] G. Floquet, Sur les equations differentielles lineaires a coefficients periodiques, Ann. de l'Ecole Norm. Suppl. 12, 47 (1883).

[20] W. Yao, A. H. MacDonald, and Q. Niu, Optical Control of Topological Quantum Transport in Semiconductors, Phys. Rev. Lett. 99, 047401 (2007).

[21] T. Kitagawa, E. Berg, M. Rudner, and E. Demler, Topological characterization of periodically driven quantum systems, Phys. Rev. B 82, 235114 (2010).

[22] N. H. Lindner, G. Refael, and V. Galitski, Floquet topological insulator in semiconductor quantum wells, Nat. Phys. 7, 490 (2011).

[23] T. Oka and H. Aoki, Photovoltaic Hall effect in graphene, Phys. Rev. B 79, 081406(R) (2009).

[24] G. Usaj, P. M. Perez-Piskunow, L. E. F. Foa Torres, and C. A. Balseiro, Irradiated graphene as a tunable Floquet topological insulator, Phys. Rev. B 90, 115423 (2014).

[25] T. Kitagawa, T. Oka, A. Brataas, L. Fu, and E. Demler, Transport properties of nonequilibrium systems under the application of light: Photoinduced quantum Hall insulators without Landau levels, Phys. Rev. B 84, 235108 (2011).

[26] N. H. Lindner, D. L. Bergman, G. Refeal, and V. Galitski, Topological Floquet spectrum in three dimensions via a two-photon resonance, Phys. Rev. B 87, 235131 (2013).

[27] Y. H. Wang, H. Steinberg, P. Jarillo-Herrero, and N. Gedik, Observation of Floquet-Bloch states on the surface of a topological insulator, Science 342, 453 (2013).

[28] J. W. McIver, B. Schulte, F.-U. Stein, T. Matsuyama, G. Jotzu, G. Meier, and A. Cavalleri, Light-induced anomalous Hall effect in graphene, Nat. Phys. 16, 38 (2019).

[29] P. M. Perez-Piskunow, G. Usaj, C. A. Balseiro, and L. E. F. Foa Torres, Floquet chiral edge states in graphene, Phys. Rev. B 89, 121401(R) (2014).

[30] W. Berdanier, M. Kolodrubetz, R. Vasseur, and J. E. Moore, Floquet Dynamics of Boundary-Driven Systems at Criticality, Phys. Rev. Lett. 118, 260602 (2017).

[31] Z. Gu, H. A. Fertig, D. P. Arovas, and A. Auerbach, Floquet Spectrum and Transport through an Irradiated Graphene Ribbon, Phys. Rev. Lett. 107, 216601 (2011).

[32] M. A. Sentef, M. Claassen, A. F. Kemper, B. Moritz, T. Oka, J. K. Freericks, and T. P. Devereaux, Theory of pump-probe photoemission in graphene: Ultrafast tuning of Floquet bands and local pseudospin textures, Nat.Commun. 6, 7047 (2015).

[33] J. Klinovaja, P. Stano, and D. Loss, Topological Floquet Phases in Driven Coupled Rashba Nanowires, Phys. Rev. Lett. 116, 176401 (2016). 
[34] J. Liu, K. Hejazi, and L. Balents, Floquet Engineering of Multiorbital Mott Insulators: Applications to Orthorhombic Titanates, Phys. Rev. Lett. 121, 107201 (2018).

[35] M. S. Rudner and N. H. Lindner, Floquet topological insulators: from band structure engineering to novel nonequilibrium quantum phenomena, Nat. Rev. Phys. 2, 229 (2020).

[36] M. Vogl, M. Rodriguez-Vega, and G. A. Fiete, Floquet engineering of interlayer couplings: Tuning the magic angle of twisted bilayer graphene at the exit of a waveguide, Phys. Rev. B 101, 241408 (2020).

[37] G. E. Topp, G. Jotzu, J. W. McIver, L. Xian, A. Rubio, and M. A. Sentef, Topological Floquet engineering of twisted bilayer graphene, Phys. Rev. Research 1, 023031 (2019).

[38] D. H. Dunlap and V. M. Kenkre, Dynamic localization of a charged particle moving under the influence of an electric field, Phys. Rev. B 34, 3625 (1986).

[39] S. Carr, S. Fang, Z. Zhu, and E. Kaxiras, Exact continuum model for low-energy electronic states of twisted bilayer graphene, Phys. Rev. Research 1, 013001 (2019).

[40] M. Koshino, N. F. Q. Yuan, T. Koretsune, M. Ochi, K. Kuroki, and L. Fu, Maximally Localized Wannier Orbitals and the Extended Hubbard Model for Twisted Bilayer Graphene, Phys. Rev. X 8, 031087 (2018).

[41] Y. Zhang, D. Mao, and T. Senthil, Twisted Bilayer Graphene Aligned with Hexagonal Boron Nitride: Anomalous Hall Effect and a Lattice Model, Phys. Rev. Research 1, 033126 (2019)

[42] Z. Song, Z. Wang, W. Shi, G. Li, C. Fang, and B. A. Bernevig, All Magic Angles in Twisted Bilayer Graphene are Topological, Phys. Rev. Lett. 123, 036401 (2019).

[43] P. Lucignano, D. Alfé, V. Cataudella, D. Ninno, and G. Cantele, Crucial role of atomic corrugation on the flat bands and energy gaps of twisted bilayer graphene at the magic angle, Phys. Rev. B 99, 195419 (2019).

[44] G. S. Uhrig, M. H. Kalthoff, and J. K. Freericks, Positivity of the Spectral Densities of Retarded Floquet Green Functions, Phys. Rev. Lett. 122, 130604 (2019).

[45] M. S. Rudner, N. H. Lindner, E. Berg, and M. Levin, Anomalous Edge States and the Bulk-Edge Correspondence for Periodically Driven Two-Dimensional Systems, Phys. Rev. X 3, 031005 (2013).

[46] P. Titum, E. Berg, M. S. Rudner, G. Refael, and N. H. Lindner, Anomalous Floquet-Anderson Insulator as a Nonadiabatic Quantized Charge Pump, Phys. Rev. X 6, 021013 (2016).

[47] H. Yoo, R. Engelke, S. Carr, S. Fang, K. Zhang, P. Cazeaux, S. H. Sung, R. Hovden, A. W. Tsen, T. Taniguchi, K. Watanabe, G.-C. Yi, M. Kim, M. Luskin, E. B. Tadmor, E. Kaxiras, and P. Kim, Atomic and electronic reconstruction at the van der
Waals interface in twisted bilayer graphene, Nature Mater. 18, 448 (2019).

[48] S. Fang, S. Carr, Z. Zhu, D. Massatt, and E. Kaxiras, AngleDependent $A b$ initio Low-Energy Hamiltonians for a Relaxed Twisted Bilayer Graphene Heterostructure, arXiv:1908.00058.

[49] E. Malic, T. Winzer, E. Bobkin, and A. Knorr, Microscopic theory of absorption and ultrafast many-particle kinetics in graphene, Phys. Rev. B 84, 205406 (2011).

[50] R. Kim, V. Perebeinos, and P. Avouris, Relaxation of optically excited carriers in graphene, Phys. Rev. B 84, 075449 (2011).

[51] S. A. Sato, J. W. McIver, M. Nuske, P. Tang, G. Jotzu, B. Schulte, H. Hübener, U. De Giovannini, L. Mathey, M. A. Sentef, A. Cavalleri, and A. Rubio, Microscopic theory for the light-induced anomalous Hall effect in graphene, Phys. Rev. B 99, 214302 (2019).

[52] S. A. Sato, P. Tang, M. A. Sentef, U. De Giovannini, H. Hübener, and A. Rubio, Light-induced anomalous Hall effect in massless Dirac fermion systems and topological insulators with dissipation, New J. Phys. 21, 093005 (2019).

[53] M. Nuske, L. Broers, B. Schulte, G. Jotzu, S. A. Sato, A. Cavalleri, A. Rubio, J. W. McIver, L. Mathey, Floquet dynamics in light-driven solids, arXiv:2005.10824.

[54] K. I. Seetharam, C. E. Bardyn, N. H. Lindner, M. S. Rudner, and G. Refael, Steady states of interacting Floquet insulators, Phys. Rev. B 99, 014307 (2019).

[55] I. Esin, G. K. Gupta, E. Berg, M. S. Rudner, and N. H. Lindner, Electronic Floquet Liquid Crystals, arXiv:2007.07909.

[56] T. Iadecola, T. Neupert, and C. Chamon, Occupation of topological Floquet bands in open systems, Phys. Rev. B 91, 235133 (2015).

[57] H. Dehghani, T. Oka, and A. Mitra, Dissipative Floquet topological systems, Phys. Rev. B 90, 195429 (2014).

[58] I. Esin, M. S. Rudner, and N. H. LindnerFloquet metal-toinsulator phase transitions in semiconductor nanowires, Sci. Adv. 6, eaay4922 (2020).

[59] Y. Li, H. A. Fertig, and B. Seradjeh, Floquet-Engineered Topological Flat Bands in Irradiated Twisted Bilayer Graphene, arXiv:1910.04711.

[60] G. Catarina, B. Amorim, E. V. Castro, J. M. V. P. Lopes, and N. M. R. Peres, in Handbook of Graphene, edited by M.i Zhang (Wiley, Hoboken, J, 2019), Vol. 3, Chap. 6, pp. 177-230.

[61] P. Delplace, Á. Gómez-León, and G. Platero, Merging of Dirac points and Floquet topological transitions in ac-driven graphene, Phys. Rev. B 88, 245422 (2013).

[62] Y.-H. Zhang, D. Mao, Y. Cao, P. Jarillo-Herrero, and T. Senthil, Nearly flat Chern bands in moire superlattices, Phys. Rev. B 99, 075127 (2019).

[63] T. Fukui, Y. Hatsugai, and H. Suzuki, Chern numbers in discretized brillouin zone: Efficient method of computing (Spin) hall conductances, J. Phys. Soc. Jpn. 74, 1674 (2005). 